\title{
Journal of Fundamental and Applied Sciences
}

ISSN 1112-9867

Available online at

http://www.jfas.info

\section{STUDY OF FLUORIDE GLASSES DEVITRIFICATION-BASED MAGNESIUM}

\author{
C. Benhamideche ${ }^{1 *}$, A. Boutarfaia ${ }^{2}$, M. Poulain ${ }^{3}$ \\ ${ }^{1}$ Faculté des Sciences, Département des sciences de la matière, Université de Skikda, Algérie. \\ ${ }^{2}$ Laboratoire de Chimie Appliquée, Université de Biskra, BP 145, 07000, Algérie. \\ ${ }^{3}$ Laboratoire de Matériaux Photoniques, Université de Rennes 1, F-35042 Rennes, France
}

Received: 4 March 2013 / Accepted: 01 April 2013 / Published online: 30 June 2013

\begin{abstract}
The kinetics of devitrification of fluoride glasses stabilized by magnesium fluoride when heated for some time between the glass transition and melting temperatures. The crystallization kinetics of $\mathrm{AlF}_{3}-\mathrm{YF}_{3}-\mathrm{PbF}_{2}-\mathrm{CdF}_{2}-\mathrm{MgF}_{2}$ glass prepared by melting the halide powders were studied by differential scanning calorimetry (DSC). The heating rate varied between 2 and $10 \mathrm{Kmim}^{-1}$. Arami's exponent $(\mathrm{n})$ obtained by a non-isothermal method was 1.5 and 2.5 for a $\mathrm{AlF}_{3}-\mathrm{YF}_{3}-\mathrm{PbF}_{2}-$ $\mathrm{CdF}_{2}-\mathrm{xMgF}_{2}(\mathrm{x}=0,5,10)$ and $\mathrm{n}>2.5$ for a $\mathrm{AlF}_{3}-\mathrm{YF}_{3}-\mathrm{PbF}_{2}-\mathrm{CdF}_{2}-15 \mathrm{MgF}_{2}$. According to the classical interpretation of $\mathrm{n}$, these magnitudes correspond to diffusion controlled growth of crystals. The activation energies for crystallization, (E), varied from 195-326 KJ/mol. These results confirm the improved stability of these glasses, which could be used as base glasses for low phonon energy fluoride glass fibers.
\end{abstract}

Keys words: crystallization kinetics, glass transition, DSC, Avrami's exponent (n), activation energies (E).

\section{INTRODUCTION}

D'après la définition du verre donnée par A. S. T. M : le verre est un produit de fusion des composés chimiques inorganiques.

Author Correspondence, e-mail: Chahrazedb2002@yahoo.fr

ICID: 1042344 
La solidification apparaît sans intervention de cristallisation, le problème traité est celui de la cristallisation d'un verre qui n'est pas une transformation qui se produit d'emblée dans tout le volume. Elle débute et s'étend progressivement à partir des centres discrets disséminés dans la masse. La connaissance des paramètres de dévitrification constitue donc un élément important pour la prévention des phénomènes de cristallisation. Elles ont ouvert à de nombreuses applications dans les domaines de la pyrométrie, de l'instrumentation et des capteurs à fibres optiques [1-2]. Le comportement de cristallisation des verres fluorés a fait l'objet de diverses études [3-5]. Notre étude s'est concentrée sur les verres appartenant à des familles dérivées du système $\mathrm{AlF}_{3}-\mathrm{YF}_{3}-\mathrm{PbF}_{2}-\mathrm{CdF}_{2}-\mathrm{MgF}_{2}$, les compositions choisies pour cette étude, les acronymes et leurs caractéristiques physiques sont affichés dans le tableau 1 [6]. Nous nous sommes donc appliqués à déterminer, par la méthode non isotherme, les principaux paramètres de la dévitrification : l'exposant d'Avrami (n) et l'énergie d'activation (E).

Tableau 1. Composition des échantillons étudiés

\begin{tabular}{ccccc}
\hline Acronyne & Composition & $\mathrm{T}_{\mathrm{g}}\left({ }^{\circ} \mathrm{C}\right)$ & $\mathrm{T}_{\mathrm{x}}\left({ }^{\circ} \mathrm{C}\right)$ & $\mathrm{T}_{\mathrm{p}}\left({ }^{\circ} \mathrm{C}\right)$ \\
\hline $\mathrm{APCYMg00}$ & $30 \mathrm{AlF}_{3}-30 \mathrm{PbF}_{2}-30 \mathrm{CdF}_{2}-00 \mathrm{MgF}_{2}-10 \mathrm{YF}_{3}$ & 337 & 476 & 495 \\
$\mathrm{APCYMg05}$ & $30 \mathrm{AlF}_{3}-30 \mathrm{PbF}_{2}-25 \mathrm{CdF}_{2}-05 \mathrm{MgF}_{2}-10 \mathrm{YF}_{3}$ & 345 & 453 & 470 \\
$\mathrm{APCYMg} 10$ & $30 \mathrm{AlF}_{3}-30 \mathrm{PbF}_{2}-20 \mathrm{CdF}_{2}-10 \mathrm{MgF}_{2}-10 \mathrm{YF}_{3}$ & 350 & $443-456$ & $451-460$ \\
$\mathrm{APCYMg} 15$ & $30 \mathrm{AlF}_{3}-30 \mathrm{PbF}_{2}-15 \mathrm{CdF}_{2}-15 \mathrm{MgF}_{2}-10 \mathrm{YF}_{3}$ & 358 & 447 & 455 \\
\hline
\end{tabular}

\section{Relation de base}

Les phénomènes de nucléation et de croissance cristalline peuvent être exprimés par de multiples relations qui dérivent d'approches théoriques. La relation définie par AVRAMI [7-9], qui est la base de nombreuses études, est la suivante:

$$
\mathrm{x}=1-\exp \left[(-\mathrm{Kt})^{\mathrm{n}}\right]
$$

Elle définit la recristallisation du verre en fonction du temps à une température donnée avec : 
$\mathrm{x}$ : fraction cristallisée (sans dimension)

$\mathrm{t}:$ temps (s)

$\mathrm{K}$ : facteur global qui tient compte des vitesses de nucléation et de croissance

$\mathrm{n}$ : coefficient d'Avrami qui dépend du mécanisme de cristallisation

Le paramètre $\mathrm{K}$ dépend de la température dans un petit domaine de température. Sa dépendance suit une loi que l'on prend habituellement de type Arrhenius:

$$
\mathrm{K}=\mathrm{K}_{0} \exp (-\mathrm{E} / \mathrm{RT})
$$

avec $\quad \mathrm{K}_{0}$ : facteur de fréquence $\left(\mathrm{s}^{-1}\right)$

E : énergie d'activation apparente décrivant l'ensemble du processus de recristallisation $\left(\mathrm{kJ} \mathrm{mol}{ }^{-1}\right)$

$\mathrm{R}$ : constante des gaz parfaits $\left(\mathrm{Pa} \mathrm{m}^{3} \mathrm{~mol}^{-1} \mathrm{~K}^{-1}\right)$

$\mathrm{T}$ : température absolue $(\mathrm{K})$

Pour déterminer l'exposant d'AVRAMI n, nous avons utilisé la méthode proposée par OZAWA appliquée aux études non isothermes. Dans cette méthode, on étudie l'évolution du pic de cristallisation d'un verre selon différentes lois de chauffe. Le taux de cristallisation à une température donnée est à chaque fois déterminé.

OZAWA pose :

$$
\mathrm{T}=\mathrm{T}_{0}+\alpha \mathrm{t}
$$

$\mathrm{T}_{0}$ : température initiale. On prend généralement $\mathrm{T}_{0}$ proche de $\mathrm{Tg}$.

$\alpha$ : loi de chauffe

a partir de (3), on écrit:

$$
\mathrm{t}=\left(\mathrm{T}-\mathrm{T}_{0}\right) / \alpha
$$

En reportant t dans l'équation (1) d'AVRAMI, on obtient :

$$
x=1-\exp \left[\frac{-K\left(T-T_{0}\right)}{\alpha}\right] n
$$

On en déduit alors en prenant deux fois le logarithme népérien :

$$
\ln [-\ln (1-x)]=n \ln \left[K\left(T-T_{0}\right)\right]-n \ln \alpha
$$


Il suffit ensuite d'enregistrer plusieurs thermogrammes $\mathrm{dH} / \mathrm{dT}=\mathrm{f}(\mathrm{T})$ pour différentes lois de chauffe $\alpha$. On représente ensuite $\operatorname{Ln}[-\operatorname{Ln}(1-x)$ ] en fonction de $\operatorname{Ln} \alpha$, la pente de cette droite nous donne la valeur du coefficient d'AVRAMI $\mathrm{n}$.

L'énergie d'activation E reposent sur l'étude de l'évolution de la position du sommet du pic de cristallisation Tp pour différentes lois de chauffe $\alpha$

La loi générale applicable est donnée par KEMENY et SESTAK [10]:

$$
|-\ln (1-x)|^{\frac{1}{n}}=\frac{k_{0} E_{a}}{R \alpha} \int_{\frac{E}{R T_{0}}}^{\infty} e^{\frac{-E_{a}}{R T}} \frac{d\left(\frac{E_{a}}{R T}\right)}{\left(\frac{E_{a}}{R T}\right)^{2}}
$$

La relation (7) conduit aux méthodes de Kissinger [11,12] et Ozawa [13,14] :

$$
\begin{aligned}
& \left\|\frac{d\left(\frac{\alpha}{T_{p}^{2}}\right)}{d\left(\frac{1}{T p}\right)}\right\|=-\frac{E_{a}}{R} \\
& \left\|\frac{d(\ln \alpha)}{d\left(\frac{1}{T p}\right)}\right\|=-\frac{E_{a}}{R}
\end{aligned}
$$

avec $\quad \mathrm{x}$ : fraction cristallisée (sans dimension)

$\mathrm{Tp}$ : température du sommet de pic de cristallisation (K)

$\alpha$ : loi de chauffe $\left(\right.$ K. $\left.\mathrm{min}^{-1}\right)$

Ea : énergie d'activation apparente décrivant l'ensemble du processus de recristallisation $\left(\mathrm{KJ} . \mathrm{mol}^{-1}\right)$

$\mathrm{R}$ : constante des gaz parfaits. 
Nous avons donc enregistré le flux thermique en fonction de la température, $\mathrm{dH} / \mathrm{dT}=\mathrm{f}(\mathrm{T})$, pour différentes lois de chauffes $\alpha, \alpha$ avec compris entre 2 et $20 \mathrm{~K} \cdot \mathrm{min}^{-1}$, et noté la température du sommet du pic de cristallisation Tp. Ensuite, il suffit de tracer les droites :

$$
\begin{array}{ll}
\operatorname{Ln}\left(\alpha / \mathrm{Tp}^{2}\right)=\mathrm{f}(1 / \mathrm{Tp}) & \text { (Kissigner) } \\
\operatorname{Ln}(\alpha)=\mathrm{f}(1 / \mathrm{Tp}) & \text { (Ozawa) }
\end{array}
$$

\section{RESULTATS}

Pour la température $\mathrm{T}=469^{\circ} \mathrm{C}$, on obtient différentes fractions cristallisées $\mathrm{x}$ selon la loi de chauffe utilisée pour chaque composition sont représentés dans le tableau 2, les courbes correspondantes sont représentées sur la figure 1.

Tableau 2. Valeurs numériques permettant de déterminer le coefficient d'Avrami n, par la méthode pseudo isotherme pour les différentes compositions.

\begin{tabular}{ccccc}
\hline Compositions & $\alpha\left({ }^{\circ} \mathrm{C} . \mathrm{min}^{-1}\right)$ & $\mathrm{Ln} \alpha$ & $\mathrm{x}$ & $\operatorname{Ln}(-\operatorname{Ln}(1-\mathrm{x}))$ \\
\hline $30 \mathrm{AlF}_{3}-30 \mathrm{PbF}_{2}-30 \mathrm{CdF}_{2}-00 \mathrm{MgF}_{2} 10 \mathrm{YF}_{3}$ & 6 & 1.7917 & 0.5716 & -0.1652 \\
& 8 & 2.0794 & 0.2875 & -1.0818 \\
$30 \mathrm{AlF}_{3}-30 \mathrm{PbF}_{2}-25 \mathrm{CdF}_{2}-05 \mathrm{MgF}_{2} 10 \mathrm{YF}_{3}$ & 10 & 2.3025 & 0.2285 & -1.3493 \\
& 6 & 2.0794 & 0.8925 & 0.8021 \\
$30 \mathrm{AlF}_{3}-30 \mathrm{PbF}_{2}-20 \mathrm{CdF}_{2}-10 \mathrm{MgF}_{2} 10 \mathrm{YF}_{3}$ & 8 & 2.3025 & 0.6080 & -0.0656 \\
& 10 & 2.9957 & 0.1756 & -1.6445 \\
$30 \mathrm{AlF}_{3}-30 \mathrm{PbF}_{2}-15 \mathrm{CdF}_{2}-15 \mathrm{MgF}_{2} 10 \mathrm{YF}_{3}$ & 8 & 1.7917 & 0.5711 & -0.1666 \\
& 6 & 2.0794 & 0.2875 & -1.0818 \\
& 10 & 2.3025 & 0.2269 & -1.3573 \\
& 6 & 2.7917 & 0.4487 & -0.5183 \\
& & 2.3025 & 0.1230 & -2.0306 \\
\hline
\end{tabular}



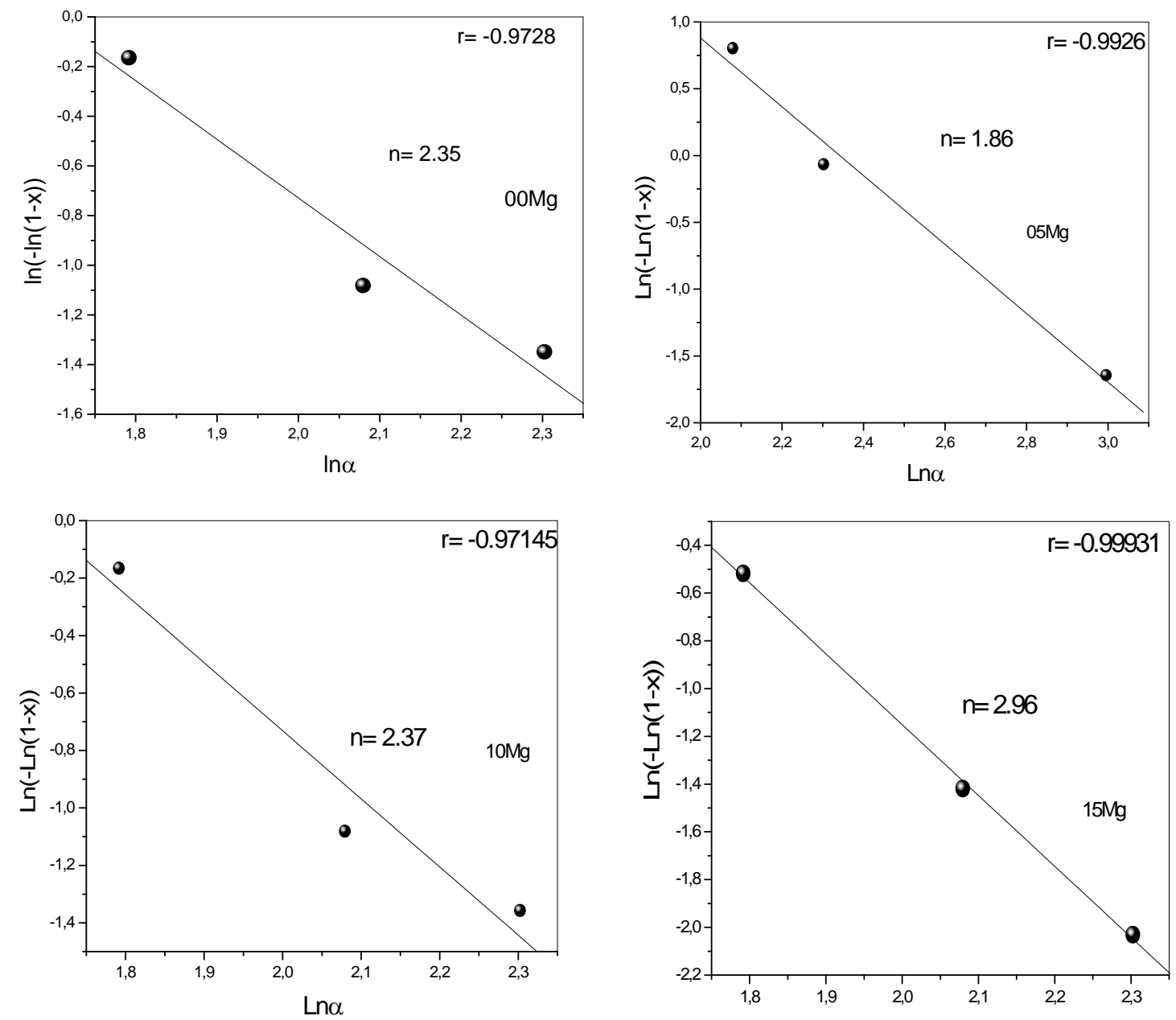

Fig.1. Détermination du coefficient d'Avrami n par la méthode pseudo isotherme pour les différentes compositions.

Le tableau 3 rassemble les différentes valeurs numériques servant à déterminer l'énergie d'activation. Les droites sont représentées sur la figure 2 . 
Tableau 3. Données numériques pour la détermination de l'énergie d'activation par les méthodes non isothermes pour les différentes compositions.

\begin{tabular}{|c|c|c|c|c|c|}
\hline Compositions & $\alpha\left(\mathrm{K} \cdot \mathrm{min}^{-1}\right)$ & $\mathrm{T}_{\mathrm{p}}(\mathrm{K})$ & $10^{3} / \mathrm{T}_{\mathrm{p}}\left(\mathrm{K}^{-1}\right)$ & $\operatorname{Ln} \alpha$ & $\operatorname{Ln}\left(\alpha / \mathrm{T}_{\mathrm{p}}^{2}\right)$ \\
\hline \multirow{3}{*}{$30 \mathrm{AlF}_{3}-30 \mathrm{PbF}_{2}-30 \mathrm{CdF}_{2}-00 \mathrm{MgF}_{2} 10 \mathrm{YF}_{3}$} & 2 & 712 & 1.404 & 0.693 & -12.443 \\
\hline & 4 & 721 & 1.386 & 1.386 & -11.774 \\
\hline & 6 & 726 & 1.377 & 1.791 & -11.383 \\
\hline \multirow{9}{*}{$30 \mathrm{AlF}_{3}-30 \mathrm{PbF}_{2}-25 \mathrm{CdF}_{2}-05 \mathrm{MgF}_{2} 10 \mathrm{YF}_{3}$} & 8 & 729 & 1.371 & 2.079 & -11.103 \\
\hline & 10 & 733 & 1.364 & 2.302 & -10.891 \\
\hline & 20 & 742 & 1.347 & 2.995 & -10.222 \\
\hline & 2 & 712 & 1.404 & 0.693 & -12.443 \\
\hline & 4 & 721 & 1.386 & 1.386 & -11.774 \\
\hline & 6 & 726 & 1.377 & 1.791 & -11.383 \\
\hline & 8 & 729 & 1.371 & 2.079 & -11.103 \\
\hline & 10 & 733 & 1.364 & 2.302 & -10.891 \\
\hline & 20 & 742 & 1.347 & 2.995 & -10.222 \\
\hline \multirow{3}{*}{$30 \mathrm{AlF}_{3}-30 \mathrm{PbF}_{2}-20 \mathrm{CdF}_{2}-10 \mathrm{MgF}_{2} 10 \mathrm{YF}_{3}$} & 2 & 712 & 1.404 & 0.693 & -12.443 \\
\hline & 4 & 724 & 1.381 & 1.386 & -11.783 \\
\hline & 6 & 733 & 1.364 & 1.791 & -11.402 \\
\hline \multirow{9}{*}{$30 \mathrm{AlF}_{3}-30 \mathrm{PbF}_{2}-15 \mathrm{CdF}_{2}-15 \mathrm{MgF}_{2} 10 \mathrm{YF}_{3}$} & 8 & 740 & 1.351 & 2.079 & -11.133 \\
\hline & 10 & 743 & 1.345 & 2.302 & -10.918 \\
\hline & 20 & 762 & 1.312 & 2.995 & -10.276 \\
\hline & 2 & 705 & 1.418 & 0.693 & -12.423 \\
\hline & 4 & 716 & 1.396 & 1.386 & -11.761 \\
\hline & 6 & 720 & 1.388 & 1.791 & -11.366 \\
\hline & 8 & 724 & 1.381 & 2.079 & -11.090 \\
\hline & 10 & 728 & 1.373 & 2.302 & -10.878 \\
\hline & 20 & 736 & 1.358 & 2.995 & -10.206 \\
\hline
\end{tabular}



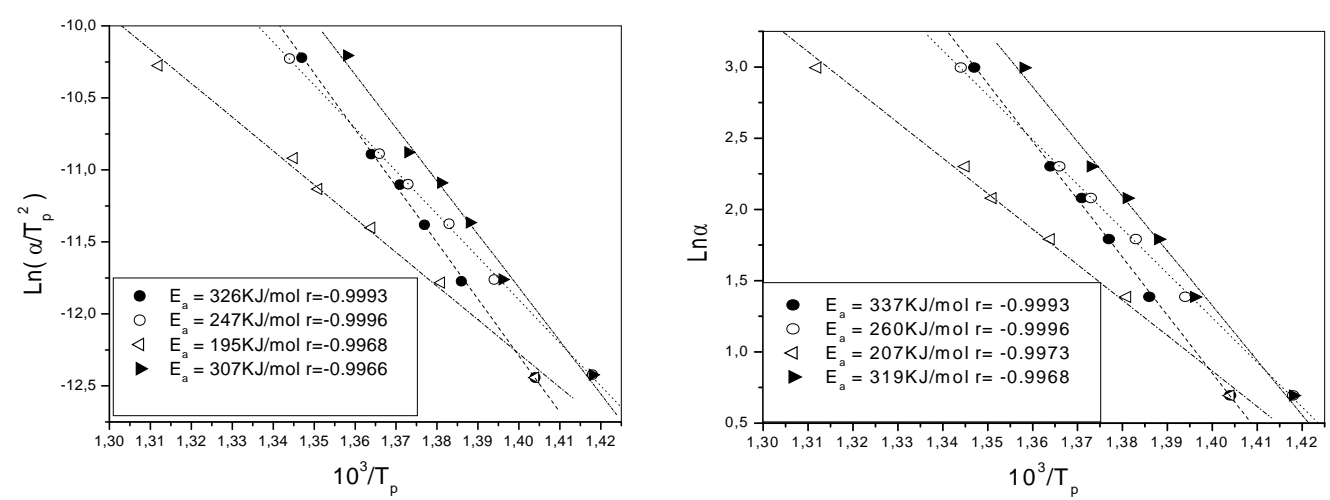

Fig.2. Détermination de l'énergie d'activation apparente de cristallisation selon la méthode de Kissinger et Ozawa.

\section{DISCUSSION}

Bien qu'il n'existe pas de règle absolue, on observe fréquemment que les verres les plus stables présentent un " $n$ "faible. Cette faible valeur de $n$ apparaît donc comme un indice probable de bonne stabilité.

On distingue habituellement deux situations pour le mécanisme de croissance selon qu'elle est contrôlée par la diffusion (CCD) ou que la vitesse de croissance de l'interface liquide / cristal est monotone : c'est alors la croissance contrôlée par l'interface (CCI) [15]. L'augmentation de n traduit une accélération de la cinétique de nucléation. Ceci suggère que le fibrage sera plus difficile, d'ailleurs il apparait les verres présentant une valeur de n élevée par exemple supérieure à 4 , sont difficiles à voir mêmes impossibles à fibrer. Dans notre cas, les valeurs de $\mathrm{n}$ pour les verres $\quad 00 \mathrm{Mg}, \quad 05 \mathrm{Mg}$ et $10 \mathrm{Mg}$ sont comprises entre 1.5 et 2.5. On se situé donc dans un schéma de croissance cristalline tridimensionnelle contrôlé par la diffusion avec un taux de nucléation décroissant au cours de la dévitrification. Cela correspondant à une température plus proche du liquide de la transition vitreuse, c'est-à-dire à un écart assez grand entre la température de cristallisation et celle de transition vitreuse, écart lui-même corrélé à la stabilité du verre. Le verre de $30 \mathrm{AlF}_{3}-30 \mathrm{PbF}_{2}-15 \mathrm{CdF}_{2}-15 \mathrm{MgF}_{2}-10 \mathrm{YF}_{3}$ présente une valeur de $\mathrm{n}>2.5$ montrant que les phénomènes de cristallisation qui suivent un schéma de croissance cristalline tridimensionnelle contrôlé par l'interface avec un taux de nucléation croissant. 
La valeur de l'énergie d'activation constitue le second paramètre cinétique majeur. Bien qu'il soit difficile de lui assigner une signification physique absolue, il est généralement admis que cette énergie d'activation est la même que celle qui régit l'évolution de la viscosité avec la température. Les résultants obtenus par les deux méthodes sont en accord. Les valeurs des énergies d'activation $E_{a}$ calculée par la méthode d'Ozawa sont légèrement supérieures pour l'ensemble des verres étudiés. Les valeurs de l'énergie d'activation $\mathrm{E}_{\mathrm{a}}$ obtenues sont situées dans la fourchette195-326 KJ/mol, ces valeurs semblent refléter une tendance à la dévitrification comparable à celle du verre standard ZBLAN [16] $(197 \mathrm{KJ} / \mathrm{mol})$ et inférieure à celle du verre ZBLA [17] (397KJ/mol). De nombreuses études ont montré que plus l'énergie d'activation apparente d'un verre est faible, plus il est stable [18]. L'écart entre les deux valeurs n'est peut être pas seulement dû aux approximations théoriques et aux incertitudes expérimentales. En effet, on sait que l'évolution de la viscosité ne suit pas une loi d'Arrhenius et donc que son énergie d'activation varie suivant la température, atteignant des valeurs élevées au voisinage de $\mathrm{T}_{\mathrm{g}}$ et des valeurs faibles vers le liquidus. Or, dans la méthode non isotherme, la cristallisation survient à des températures plus élevées; il est donc logique que la valeur de l'énergie d'activation apparente soit abaissée. Donc l'incorporation de $\mathrm{Mg}$ dans la matrice de base conduit à une diminution de l'énergie d'activation et dont l'augmentation de la stabilité du verre à un certains pourcentage du fluorure de magnésium et remonte rapidement; ce dernier point est intéressant car la différence observée de $195 \mathrm{KJ} / \mathrm{mol}$ à $307 \mathrm{KJ} / \mathrm{mol}$ exprime un accroissement de la stabilité du verre qui correspond aux observations expérimentales, ces résultats sont représentés graphiquement dans la figure 3. 


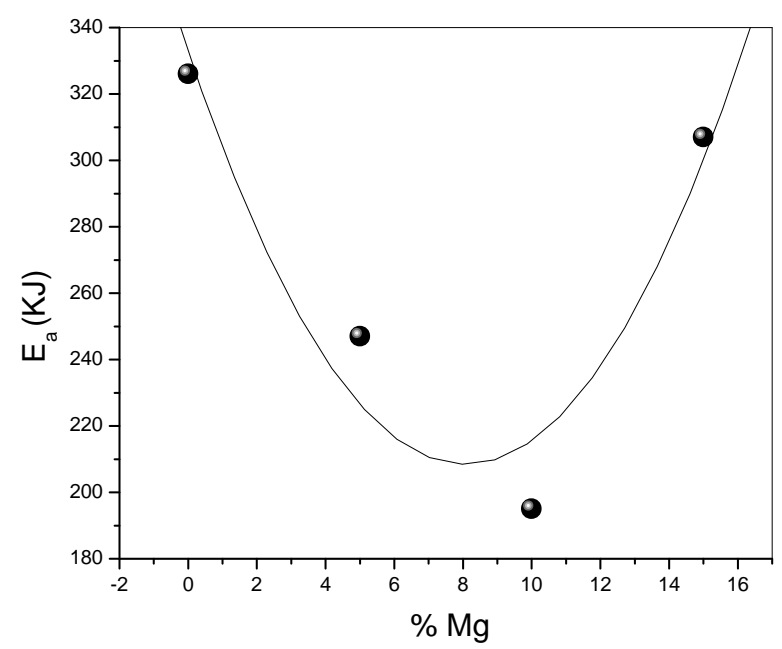

Fig.3. Evolution de l'énergie d'activation en fonction du $\% \mathrm{MgF}_{2}$

\section{CONCLUSION}

La dévitrification de quatre verres de fluoroaluminates de formulation complexe a été étudiée en utilisant des procédures non isothermes. On a ainsi pu déterminer la valeur de l'exposant d'Av rami $\mathrm{n}$ et celle de l'énergie d'activation apparente $\mathrm{E}$ à la cristallisation. Les valeurs de $\mathrm{n}$ inférieures à 3 et celles d'E sont cohérentes avec l'assez bonne résistance de ces verres à la recristallisation lors de l'étape de coulée et de solidification. Ils apparaissent donc propices à des essais d'élaboration de préformes et de fibres optiques.

\section{REFERENCES}

[1] Poulain M., Poulain M., Riv. Della Staz. Sper. Vetro, 1989 (19), 107.

[2] Tioua B., Soltani M. T., Hamzaoui M., Baazouzi M. J. Fund. App. Sci., 2012, 4(1) 1-10.

[3] Babai A., Aiadi K. E., Bentouila O., Rehoma F. J. Fund. App. Sci., 2012, 4(1) 57-65.

[4] Matecki M., Poulain M., Izumitani T., Mat. Sci. For., 1991, (67-68), 233.

[5] M. Poulain, J. Non-Cryst. Solids, 1992, (140), 1.

[6] Benhamidéche C., Boutarfaia A., Poulain M., J. Alloys Compounds., 2004, (366) 233.

[7] Avrami M., J. Phys. Chem., 1939 (7) 1103.

[8] M. Avrami, J. Phys. Chem., 1940 (1 8) 212.

[9] Avrami M., J. Phys. Chem., 1941 (9) 177. 
[10] Kemeny T., Sestak J., Thermochimica Acta., 1987 (110) 113.

[11] Kissinger H. E., J. Res. Nat. Bur. Stand, 1956 (57) 217.

[12] Kissinger H. E., Anal. Chem., 1957 (29) 1702.

[13] Ozawa T., Bull. Chem. Soc. Japan., 1965 (38) 188.

[14] Ozawa T., Polymer., 1971 (12) 150.

[15] Christian J. W. "The theory of transformation in metals and alloys" $2^{\text {nd }}$ Ed., Pergamon, New York 1971.

[16] Lecoq A., Poulain M., J. Non-Cryst. Solids., 1981 (44) 209.

[17] Smektala F., Thèse de doctorat, université de Rennes 11991.

[18] Moynihan C. T., Gavin D. L., Chang K. H., Bruce A. T., Drexhage M. G., El Bayoumi O. H., Glastech. Ber. 1983 (56) 862.

\section{How to cite this article}

Benhamideche C, Boutarfaia A and Poulain M. Study of fluoride glasses devitrification-based magnesium. J Fundam Appl Sci. 2013, 5(1), 14-24. 